\title{
Development of Nanoscale Thermal Properties Measurement Technique by Using Near-Field Optics*
}

\author{
Yoshihiro TAGUCHI ${ }^{* *}$, Yukihiro HORIGUCHI ${ }^{* *}$, Mikako KOBAYASHI**, \\ Toshiharu SAIKI** and Yuji NAGASAKA**
}

\begin{abstract}
The nanoscale thermal properties are becoming increasingly important for the thermal design of electronic devises as the MEMS technology makes progress. The thermal conductivity of nanoscale thin film is remarkably lower than that of bulk materials because of its various size effects. In addition, the nano-materials will have pointing defects or lattice imperfections during the production process. Therefore, nanoscale thermal properties measurement technique, which can be applied in-situ or in-process, is required. We have developed a new thermal properties measurement technique by using near-field optics, which targets spatial resolution better than $100 \mathrm{~nm}$ (up to $10 \mathrm{~nm}$ ) and is applicable to measure the thermal properties of nanoscale materials in-situ. In this article, in order to check the validity of the control system, the topographic image of diffraction grating is monitored. Moreover, the temperature change of $\mathrm{Al}$ thin film is detected as a thermoreflectance signal. Finally, the capability of our present work, to measure the thermal properties of nanostructures, is discussed.
\end{abstract}

Key Words: Near-Field Optics, Thermal Conductivity, Thermal Diffusivity, Thermoreflectance

\section{Introduction}

In the field of nanotechnology, nanoscale thermal properties of semiconductor devices such as ULSI or nano-materials (e.g. carbon nano tube and nano wire etc.) are required for the numerical simulation of thermal design. Especially, in mesoscopic scale where the representative length is comparable to the mean free path of material, various size effects (i.e. scattering of heat carriers by interfacial boundaries, lattice imperfections and grain boundaries, etc.) may affect the difference of thermal properties between thin film and bulk material ${ }^{(1)}$. Akabori et al. ${ }^{(2)}$ have presented the measurements of the thermal diffusivity of sputtered $\mathrm{Ti}$ and $\mathrm{Zr}$ thin films by using the photoacoustic method, and have found that the thermal diffusivities of those films are less than half of the corresponding bulk values. Cahill et al. ${ }^{(3)}$ have measured nanometer-thick layers of oxides, and have suggested the decrease of thermal conductivity caused by the effect of in-

* Received 21st January, 2004 (No. 04-4032)

** School of Integrated Design Engineering, Keio Univ., 314-1 Hiyoshi, Yokohama, Kanagawa 223-8522, Japan. E-mail: tag@naga.sd.keio.ac.jp, hori@naga.sd.keio.ac.jp, mikako@naga.sd.keio.ac.jp, saiki@elec.keio.ac.jp, nagasaka@sd.keio.ac.jp terfaces on heat transport. Graebner et al. ${ }^{(4)}$ have worked on several thicknesses of CVD polycrystalline diamond films, and have reported the film thickness dependence of thermal conductivity. As mentioned above, it is necessary to measure the thermal properties of thin films or nanomaterials for accurate numerical simulation of integrated semiconductor devices and for understanding nanoscale heat transfer of materials. Moreover, in its fabrication process, thin film or nano-material may have nanostructural difference from corresponding bulk material or interfacial conditions, thus in-situ measurement technique for the inprocess control is required.

In the last decade, several different techniques have been developed to measure and predict the thermal properties of thin films and nano-materials (see Table 1). Although the $3 \omega$ method is remarkably suitable for measuring the thermal conductivity of ultra-thin films ${ }^{(3)}$, it is not applicable to in-situ condition because $3 \omega$ method requires the deposition of sensor unit on a sample surface. In addition, its spatial resolution is not significantly high compared to other optical techniques. The non-destructive and non-contact optical technique (picosecond thermoreflectance technique ${ }^{(5)}$ ) has been developed to measure the thermal diffusivity of thin films. However, this conventional optical thermometry method has a spatial resolu- 
Table 1 Comparison of nanoscale thermal property measurement techniques

\begin{tabular}{lclll}
\hline & Spatial Resolution & Probe Type & in-situ Application & Sample \\
\hline $3 \omega$ method [3] & $\mathrm{xy}:>10 \mu \mathrm{m}$ & contact & No & multilayer d: $10 \mathrm{~nm} \sim \mu \mathrm{m}$ \\
ps thermoreflectance technique [5] & $\mathrm{xy}:>1 \mu \mathrm{m}$ & non-contact & No & monolayer d: $>100 \mathrm{~nm}$ \\
TC-AFM [6] & $\mathrm{xy}: 10 \sim 100 \mathrm{~nm}$ & contact & Yes & monolayer d: $>\mu \mathrm{m}$ \\
Present work (target) & $\mathbf{x y :}>\mathbf{1 0} \mathbf{n m}$ & non-contact & Yes & multilayer d: $>\mathbf{1 0 0} \mathbf{n m}$ \\
\hline
\end{tabular}

tion limit (up to wavelength of light source) owing to the diffraction limit. TC-AFM ${ }^{(6)}$ has a high potential to measure nanoscale thermal imaging because of its high spatial resolution (sub-100 nm), yet its depth resolution is up to micrometer order due to its low response of thermometer.

We have developed a new thermometry for measuring the nanoscale thermal properties in-situ by using nearfield optics. The aim of this study is to measure each thermal properties of $100 \mathrm{~nm}$ layered sample with $10 \mathrm{~nm}$ spatial resolution. In this paper, we will discuss the validity of the measurement theory, and the first prototype of nanoscale thermometry by using near-field optics (NOTN: Near-field Optics Thermal Nanoscopy) to measure the nanoscale thermal properties. So as to evaluate the applicability of our experimental setup, the topographic image of diffraction grating and thermometry of Al thin film are demonstrated. Finally, the preliminary examination of new thermal properties measurement technique is reported.

\section{Nomenclature}

$\begin{aligned} a & : \text { thermal diffusivity } \\ d & : \text { sample thickness } \\ f & : \text { modulation frequency } \\ k & : \text { wave number } \\ I_{0} & : \text { incident laser light flux } \\ R & : \text { reflectivity } \\ \Delta R & : \text { reflection coefficient } \\ \Delta T & : \text { temperature change } \\ w & : \text { radius of circular aperture }\end{aligned}$

$\left[\mathrm{m}^{2} \cdot \mathrm{s}^{-1}\right]$
$[\mathrm{m}]$
$[\mathrm{Hz}]$
$\left[\mathrm{m}^{-1}\right]$
$\left[\mathrm{W} \cdot \mathrm{m}^{-2}\right]$
$[-]$
$[-]$
$[\mathrm{K}]$
$[\mathrm{m}]$
Greek

$\alpha:$ absorption coefficient

$\left[\mathrm{m}^{-1}\right]$

$\beta$ : relative reflectance temperature coefficient

$\left[\mathrm{K}^{-1}\right]$

$\varepsilon:$ dielectric constant

[-]

$\Delta \phi:$ phase-lag

$\lambda$ : thermal conductivity

$\lambda_{\mathrm{opt}}$ : wavelength

[deg.]

$\left[\mathrm{W} \cdot \mathrm{m}^{-1} \cdot \mathrm{K}^{-1}\right]$

[m]

$\omega$ : angular frequency of modulation

$\omega_{\text {opt }}:$ optical frequency

$\left[\mathrm{rad} \cdot \mathrm{s}^{-1}\right]$

$\left[\mathrm{s}^{-1}\right]$

$\left[\mathrm{s}^{-1}\right]$

$\omega_{\mathrm{c}}:$ average collision frequency

$\omega_{\mathrm{p}}$ : electron plasma frequency

Subscripts

$\left[\mathrm{s}^{-1}\right]$

$$
\begin{aligned}
& \mathrm{h}: \text { heating laser } \\
& \mathrm{s}: \text { sample } \\
& \mathrm{z}: \text { PZT modulation }
\end{aligned}
$$

\section{The Principle of Measurement}

\subsection{Near-field optics}

Figure 1 (a) shows a schematic image of near-field optical fiber that has a circular aperture with a radius $w$ ( $w$ is much smaller than the wavelength $\lambda_{\text {opt }}$ of the incident beam). In this case, a magnetic current and magnetic charge, which are inside the aperture when the probe is irradiated by the laser beam, are assumed by using the magnetic dipole and electronic dipole with normal orientation. The exact solution of this problem has been presented by Bethe $^{(7)}$. Bethe has considered a solution to Maxwell's equations for a small circular hole in a conducting thin screen for an incident wave of arbitrary polarizations. For a circular aperture of radius $w\left(2 w \ll \lambda_{\text {opt }}\right)$, the electronic field $\mathbf{E}(\mathbf{r})$ and magnetic field $\mathbf{H}(\mathbf{r})$ at the point $\mathbf{r}(x, y, z)$ are described as following equations with Green's function $G$.

$$
\begin{aligned}
& \mathbf{E}(\mathbf{r})=\int_{S}\left[\mathbf{K}\left(\mathbf{r}^{\prime}\right) \times \nabla G\right] d x d y, \\
& \mathbf{H}(\mathbf{r})=\int_{S}\left[j k \mathbf{K}\left(\mathbf{r}^{\prime}\right)-\eta \nabla G\right] d x d y .
\end{aligned}
$$

Also, the quantities with the dimensions of magnetic current density $\mathbf{K}$ and magnetic charge density $\eta$ are respectively described as,

$$
\mathbf{K}\left(\mathbf{r}^{\prime}\right)=\frac{1}{\pi^{2}}\left\{j k \sqrt{\left(w^{2}-\left|\mathbf{r}^{\prime}\right|^{2}\right)} \mathbf{H}_{0}+\frac{1}{2 \sqrt{\left(w^{2}-\left|\mathbf{r}^{\prime}\right|^{2}\right)}} \mathbf{r}^{\prime} \times \mathbf{E}_{0}\right\},
$$

$\eta\left(\mathbf{r}^{\prime}\right)=\frac{1}{\pi^{2} \sqrt{\left(w^{2}-\left|\mathbf{r}^{\prime}\right|^{2}\right)}} \mathbf{r}^{\prime} \cdot \mathbf{H}_{0}$,

where, $\mathbf{r}^{\prime}(x, y, 0)$ is distance of the source point from the origin at center of the aperture, $k$ is the wave number $\left(k=2 \pi / \lambda_{\text {opt }}\right), \mathbf{H}_{0}$ is the tangential magnetic components, and $\mathbf{E}_{0}$ is the normal electronic components. The electrical field energy density $|\mathbf{E}|^{2}$ is easily calculated by Eq. (1), and it is suggested that the near-field optics decays with distance from an aperture ${ }^{(8)}$. Because of localized distribution of magnetic field, near-field optics has a high spatial resolution $\delta$ that is dependent on the diameter of the circular aperture $(\delta \sim 2 w)$. In order to illuminate the sample by near-field optics, it is necessary to hold the tip-sample displacement within nanometer order. 


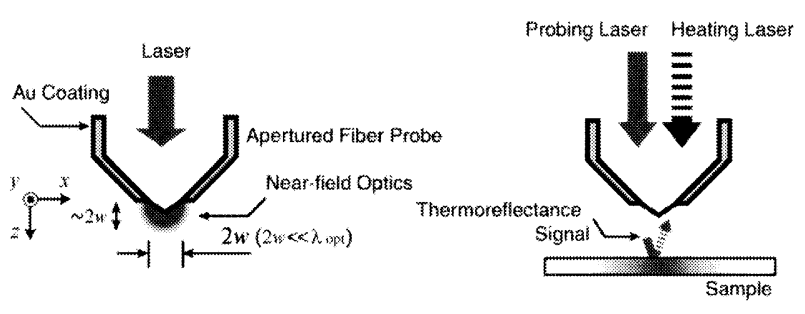

(a) A near-field optical fiber

(b) Thermoreflectance method

Fig. 1 (a) Schematic image of near-field optics. The needlepoint is coated by $\mathrm{Au}$, and has a circular aperture. (b) The power of reflected light, which is generated by near-field optics of probing beam, is depended on the temperature change of sample.

\subsection{Measurement theory of thermal properties}

3.2.1 Thermometry For measuring temperature changes of sample surface, temperature dependence of reflectivity, so-called thermoreflectance, is utilized. The reflectivity and other optical constants of metals have been discussed on the basis of the Drude theory and the theory of electron-phonon collision ${ }^{(9)}$. The electron-phonon collision frequency is dependent on the temperature, and thus the temperature dependence of the collision frequency determines the optical properties of metals and semiconductors. The reflectivity $R$ is given in terms of the complex refractive index $\varepsilon$ by

$$
\begin{aligned}
R & =\left|\frac{\sqrt{\varepsilon}-1}{\sqrt{\varepsilon}+1}\right|^{2}, \\
\varepsilon & =\varepsilon_{1}+j \varepsilon_{2} \\
& =1-\frac{\omega_{\mathrm{p}}^{2}}{\omega_{\mathrm{opt}}^{2}+\omega_{\mathrm{c}}^{2}}-j \frac{\omega_{\mathrm{p}}^{2} \omega_{\mathrm{c}}^{2}}{\left(\omega_{\mathrm{opt}}^{2}+\omega_{\mathrm{c}}^{2}\right) \omega_{\mathrm{opt}}},
\end{aligned}
$$

where $\varepsilon_{1}$ and $\varepsilon_{2}$ are real and imaginary parts of the dielectric constant respectively, $\omega_{\mathrm{c}}$ is the average collision frequency with the vibrating lattice, $\omega_{\mathrm{p}}$ is the electron plasma frequency, and $\omega_{\text {opt }}$ is the optical frequency. The reduction in the reflectivity with temperature change for $\mathrm{Al}$ is numerically calculated in Fig. 2 . In this theory, for small temperature changes, the reflection coefficient $\Delta R$ is proportional to the temperature change $\Delta T$ as follows.

$$
\Delta R=\beta \Delta T,
$$

where $\beta$ is the relative reflectance temperature coefficient, and, for the metal material, $\beta$ is estimated to be $\sim 10^{-5} \mathrm{~K}^{-1}$. By using near-field optics as a probing source, we can observe the temperature change and its distribution with high spatial resolution (see Fig. 1 (b)).

3.2.2 Periodic heating theory In order to measure the thermal conductivity and thermal diffusivity of thin film on the substrate, periodic heating theory that is based on Rosencwaig-Gersho theory (RG theory) ${ }^{(10)}$ is adopted. RG theory describes the periodic temperature distribution with photoacoustic effect. Figure 3 illustrates a schematic diagram of periodic heating theory,

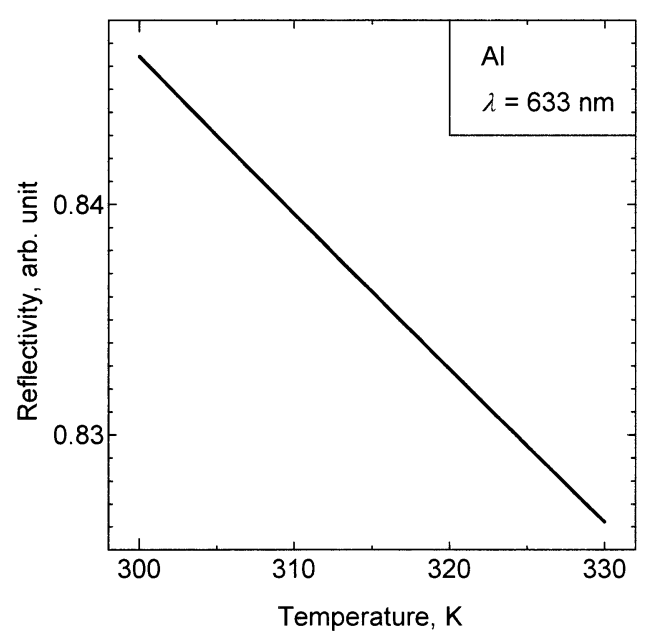

Fig. 2 Temperature dependence of reflectivity of $\mathrm{Al}$ at wavelength $\lambda_{\text {opt }}=633 \mathrm{~nm}$

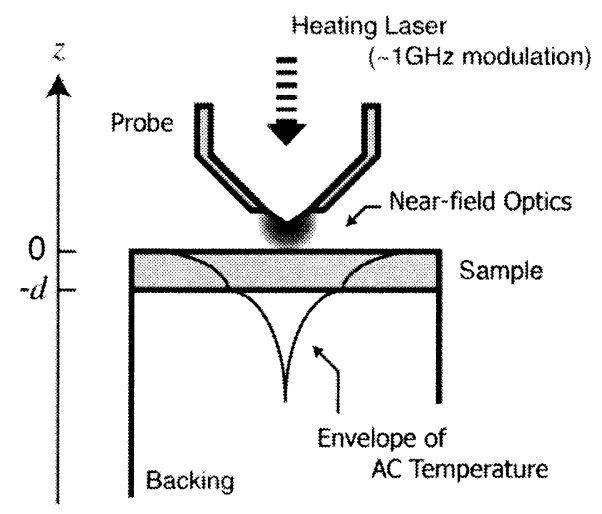

Fig. 3 The principle of NOTN. The sample surface is sinusoidally heated by near-field optics, and the temperature change due to the heating laser is detected as a change of reflectivity (thermoreflectance method).

and the $\mathrm{AC}$ temperature is derived form the RG theory for the single-layer model. When the sample surface is sinusoidally heated by near-field optics as shown in Fig. 3, the reflectivity of sample has a sinusoidal variation with temperature change (thermoreflectance signal). The thermoreflectance signal contains the information of thermal properties, sample thickness and backing properties. Thus, by analyzing the thermoreflectance signal that is sinusoidally modulated by near-field optics, we can extract the thermal conductivity and thermal diffusivity simultaneously.

The sample is heated by the near-field optics that is sinusoidally modulated at an angular frequency $\omega$ as

$$
I=\frac{I_{0}}{2}(1+\cos \omega t),
$$

where $I_{0}$ is the incident laser light flux. We assume the penetration depth $L$ for the wavelength $\lambda$ of light source, and the heat density $q$ that is produced at any point $z$ due to light absorption can be written by

$$
q=\frac{1}{2} \alpha_{\mathrm{s}} I_{0} \exp \left(\alpha_{\mathrm{s}} z\right) \times(1+\cos \omega t)
$$


where $\alpha_{\mathrm{s}}$ is a absorption coefficient $=1 / L$. In the case of metal sample, penetration depth is estimated to be about order of several nanometer so that the sample is opaque to the heating light source. Hence, the heat conduction equations in this problem are derived from the Fourier's equations as follows,

$$
\begin{aligned}
& \frac{\partial^{2} \Phi_{\mathrm{g}}}{\partial z^{2}}=\frac{1}{a_{\mathrm{g}}} \frac{\partial \Phi_{\mathrm{g}}}{\partial t} \quad(0 \leq z), \\
& \frac{\partial^{2} \Phi_{\mathrm{s}}}{\partial z^{2}}=\frac{1}{a_{\mathrm{s}}} \frac{\partial \Phi_{\mathrm{s}}}{\partial t}-\frac{\alpha_{\mathrm{s}} I_{0}}{2 \lambda_{\mathrm{s}}} \exp \left(\alpha_{\mathrm{s}} z\right) \times(1+\cos \omega t) \quad(-d \leq z \leq 0), \\
& \frac{\partial^{2} \Phi_{\mathrm{b}}}{\partial z^{2}}=\frac{1}{a_{\mathrm{b}}} \frac{\partial \Phi_{\mathrm{b}}}{\partial t} \quad(-\infty \leq z \leq-d),
\end{aligned}
$$

where $\Phi$ is the complex temperature in each layer, $a$ is the thermal diffusivity, and $\lambda$ is the thermal conductivity, respectively. To solve these heat conduction equations, the appropriate boundary conditions (the continuity of temperature and heat flux) are considered. Here the assumption of continuity conditions are

$$
\begin{aligned}
& \Phi_{\mathrm{g}}(0, t)=\Phi_{\mathrm{s}}(0, t), \quad \Phi_{\mathrm{s}}(-d, t)=\Phi_{\mathrm{b}}(-d, t), \\
& -\lambda_{\mathrm{g}} \frac{\partial \Phi_{\mathrm{g}}}{\partial z}(0, t)=-\lambda_{\mathrm{s}} \frac{\partial \Phi_{\mathrm{s}}}{\partial z}(0, t), \\
& -\lambda_{\mathrm{s}} \frac{\partial \Phi_{\mathrm{s}}}{\partial z}(-d, t)=-\lambda_{\mathrm{b}} \frac{\partial \Phi_{\mathrm{b}}}{\partial z}(-d, t), \\
& \Phi_{\mathrm{g}}(\infty, t)=0, \quad \Phi_{\mathrm{b}}(-\infty, t)=0 .
\end{aligned}
$$

Therefore, the solution for $\theta$, the complex amplitude of the periodic AC temperature $\Phi_{\mathrm{AC}}(0, t)=\theta \exp (j \omega t)$ at the solid-gas boundary $(z=0)$, is given by

$$
\begin{aligned}
\theta= & \frac{\alpha_{\mathrm{s}} I_{0}}{2 \lambda_{\mathrm{s}}\left(\alpha_{\mathrm{s}}^{2}-\sigma_{\mathrm{s}}^{2}\right)} \\
& \times \frac{(b+1)(\gamma-1) \exp \left(\sigma_{\mathrm{s}} d\right)-(b-1)(\gamma+1) \exp \left(-\sigma_{\mathrm{s}} d\right)+2(b-\gamma) \exp \left(-\alpha_{\mathrm{s}} d\right)}{(b+1)(g+1) \exp \left(\sigma_{\mathrm{s}} d\right)-(b-1)(g-1) \exp \left(-\sigma_{\mathrm{s}} d\right)},
\end{aligned}
$$

where $\sigma_{\mathrm{s}}=(1+j) \sqrt{\omega / 2 a_{\mathrm{s}}}$ is the complex wave number of temperature wave in the material, $b=e_{\mathrm{b}} / e_{\mathrm{s}}$ is the ratio of the thermal effusivity of the backing to that of the sample, $g=e_{\mathrm{g}} / e_{\mathrm{s}}$ is the ratio of the thermal effusivity of material (thermal effusivity $e=\sqrt{\rho C_{\mathrm{p}} \lambda}$ ), and $\gamma=\alpha / \sigma_{\mathrm{s}}$. The actual temperature distribution of sample surface is given by the real part of complex solution, thus, the detected variation in reflectance is written as follows,

$$
\begin{aligned}
\Delta & R_{\mathrm{AC}}=\beta \Delta T_{\mathrm{AC}}=\beta \operatorname{Re}\{\theta \exp (j \omega t)\} \\
& =\beta \operatorname{Re}\{A \exp (-j \Delta \phi) \exp (j \omega t)\} \\
& =\beta A \cos (\omega t-\Delta \phi),
\end{aligned}
$$

where $A$ and $\Delta \phi$ denote the amplitude and phase-lag between sinusoidally modulated light source and the detected light power, which depends on the reflectance of sample. The phase-lag $\Delta \phi$ is defined as

$$
\Delta \phi=\tan ^{-1}\left[\frac{-\operatorname{Im} \theta}{\operatorname{Re} \theta}\right] .
$$

In Fig. 4, for visualization of periodic heating theory, the phase-lag of various metallic samples with acrylic backing is evaluated in the function of modulated frequency (Eq. (19)). As can be seen from Fig. 4, the phaselag $\Delta \phi$ of detected signal is significantly sensitive to the variation of thermal properties of sample in high frequency region. The thermal diffusion length $\left(\mu=\sqrt{2 a_{\mathrm{s}} / \omega}\right)$ is inversely proportional to the modulated angular frequency $\omega$, consequently, when we measure the thin film in the order of a few hundreds nanometer, ultra high modulation frequency is required. By analyzing the phase-lag between the modulated heating light and detected probing light, we can determine the thermal conductivity and thermal diffusivity of sample, simultaneously.

\section{Experimental Apparatus}

A system configuration of the present experimental

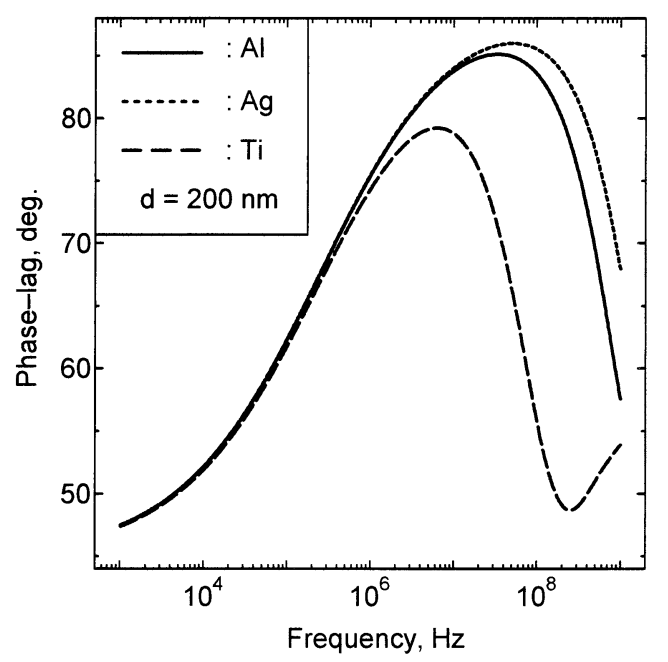

Fig. 4 RG theory in various samples on acrylic substrate. The sample thickness is $200 \mathrm{~nm}$. Variation of signal is observed in high frequency region. 


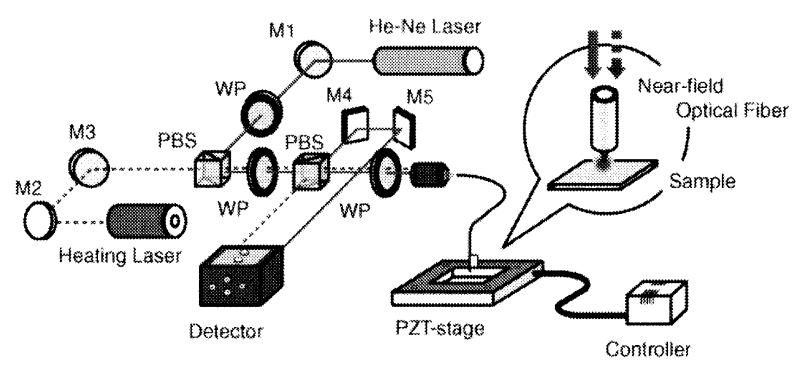

Fig. 5 Experimental apparatus of NOTN

apparatus is illustrated in Fig. 5. We have designed and constructed the control system to maintain the near-field optical fiber within the proximity region of sample. In order to observe the nanometer order displacement between the tip and sample, a shear-force (i.e. the van der Waals force, viscosity force, etc. ${ }^{(1),(12)}$ ) is adopted. This technique is already established in an atomic force microscope $(\mathrm{AFM})^{(13)}$. The fiber tip is vibrated at a resonant frequency, and the vibration amplitude is monitored as a function of tip-sample displacement. As the tip approaches the sample surface, the vibration amplitude of near-field optical fiber reduces due to the shift of the resonant frequency caused by the shear-force. Then, the feedback control of vibration amplitude allows the tip-sample displacement to be held constant. Actually, the nearfield optical fiber is attached to the quartz crystal tuningfork, and the tuning-fork modulates the fiber at resonant frequency $(\sim 30 \mathrm{kHz})$. By controlling the amplitude of tuning-fork, we can hold the tip-sample displacement in near-field region, and scan the sample surface to measure the topographic image. Furthermore, the error due to thermal expansion can be eliminated by the feedback control of tip-sample displacement.

The sample surface is illuminated by near-field optics that is induced by a He-Ne laser ( $633 \mathrm{~nm}$ wavelength and $1 \mathrm{~mW}$ optical power), and the radiation reflects back within the fiber to the detector. Furthermore, the high frequency-modulated laser (up to $1 \mathrm{GHz}$ ) generates the near-field optics at the tip of fiber as a heat source. The thermoreflectance signal is monitored by a lock-in amplifier for measuring the phase-lag versus frequency.

The characteristics of this method is summarized as follows: it is possible (1) to observe the nano-scale heat transport phenomenon (the spatial resolution is up to $10 \mathrm{~nm}$ ); (2) to evaluate the thermal properties (thermal conductivity and thermal diffusivity) simultaneously in-situ condition; and (3) to measure the multi layer thin film (up to $100 \mathrm{~nm}$-thick).

\section{Signal Detection Methodology}

For measurement of thermal properties by using nearfield optics thermal nanoscopy, there are serious problems as shown in Fig. 6. When the sample is illuminated by

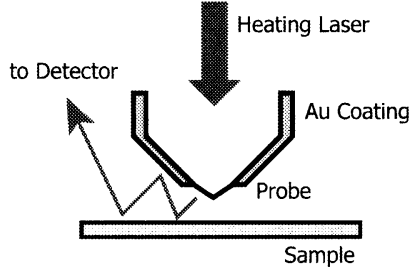

(a) Illumination mode

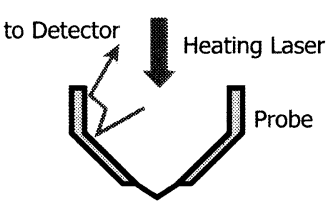

(b) Illumination-collection mode
Fig. 6 The effect of scattered light at tip of fiber. (a) Far-field detection, and (b) near-field detection

near-field optics, the thermoreflectance signal is collected by far-field objective (illumination-mode, in Fig. 6 (a)). In this case, the detected signal may contain the far-field propagation photons that are initially diffracted at the circular aperture of fiber, and this diffused reflection light will reduce the $\mathrm{S} / \mathrm{N}$ ratio. Therefore, we have employed the illumination-collection mode (the probing light reflects back within the fiber to the detector; namely, I-C mode, see Fig. 6(b)). However, in the I-C mode, the detected signal may have a background noise due to the reflection of probing laser beam from $\mathrm{Au}$ coated aperture, which is affected by the angular frequency $\omega_{\mathrm{h}}$ of modulated heating laser. In order to eliminate the background noise of the signal, we have proposed the novel detection technique Probe Oscillation Method (POM).

The near-field optics is localized as shown in section 3, so that the intensity of near-field optics decreases proportionally to the distance from circular aperture of fiber. Provided that the PZT stage is vibrated by external function $\left(\omega_{z}: 10^{1} \sim 10^{2} \mathrm{kHz}\right.$ order $)$ with respect to the $z$-axis of fiber, the intensity of heating laser is modulated as the following equation,

$$
I=\frac{I_{0}}{4}\left(1+\cos \omega_{\mathrm{h}} t\right)\left(1+\cos \omega_{\mathrm{z}} t\right) .
$$

Attending to the frequency of difference between the heating laser and PZT stage $\omega_{\mathrm{h}}-\omega_{\mathrm{z}}$, the sample is heated by its beat frequency, and the thermoreflectance signal is given by

$$
\Delta R_{\mathrm{POM}}=\beta A \cos \left\{\left(\omega_{\mathrm{h}}-\omega_{\mathrm{z}}\right) t-\Delta \phi_{\mathrm{h}}-\Delta \phi_{\mathrm{z}}\right\} .
$$

Hence, we can extract the thermoreflectance signal that includes the information of thermal properties without the influence of background noise.

\section{Results and Discussions}

\subsection{Control system and optical setup}

Verification of present apparatus is demonstrated using the diffraction grating (Nikon; $36 \mu \mathrm{m}$ wavelength, height nominal $200 \mathrm{~nm}$ ), and its topographic image is shown in Fig. 7. The iso-intensity line of shear-force traces the geometric shape of grating, and the detected topographic image well agrees with the results using commercially available AFM (cantilever-type). The roughness 


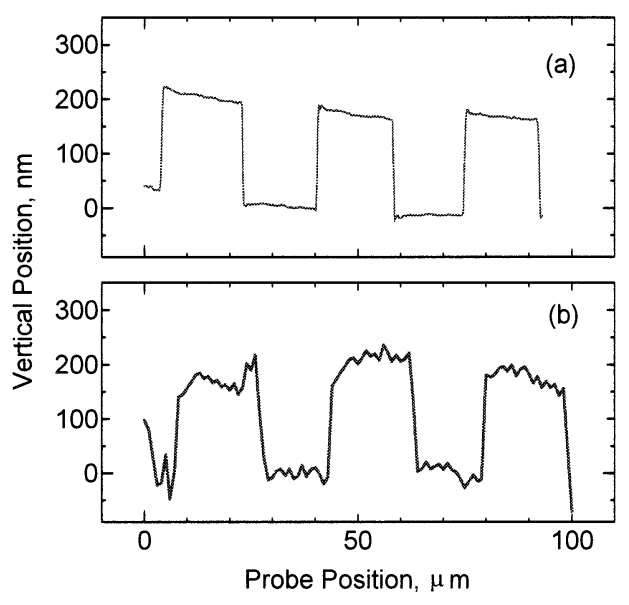

Fig. 7 The topographic image of diffraction grating. (a) by cantilever-type AFM (SII; SPM300), and (b) by the present setup

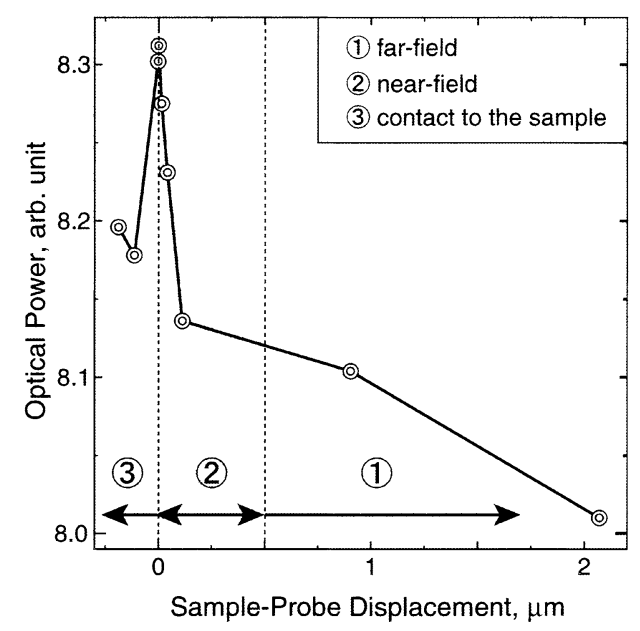

Fig. 8 The distance dependence of detected optical power. In the proximity regime, the optical power increases exponentially.

of detected topographic image is caused by the stability of control system and the $\mathrm{S} / \mathrm{N}$ ratio of detected signal. Consequently from these results, the validity of present control system is confirmed, and we can control the distance between tip of fiber and sample within nanometer order.

To confirm the adequacy of control system and optical setup for detecting a near-field optics, sample-probe distance dependence of optical power is investigated. In Fig. 8, the detected optical power versus tip-sample displacement is exhibited. In the far-field regime, the optical power increases moderately; on the other hand, the detected signal drastically increases in the near-field regime. The near-field optics, which is localized within a proximity region of the fiber tip, is scattered by the interaction between the sample surface and tip so that the intensity of detected signal increases exponentially depending on the distance from the sample. As a result of this, our optical system has a validity to measure the weak optical signal

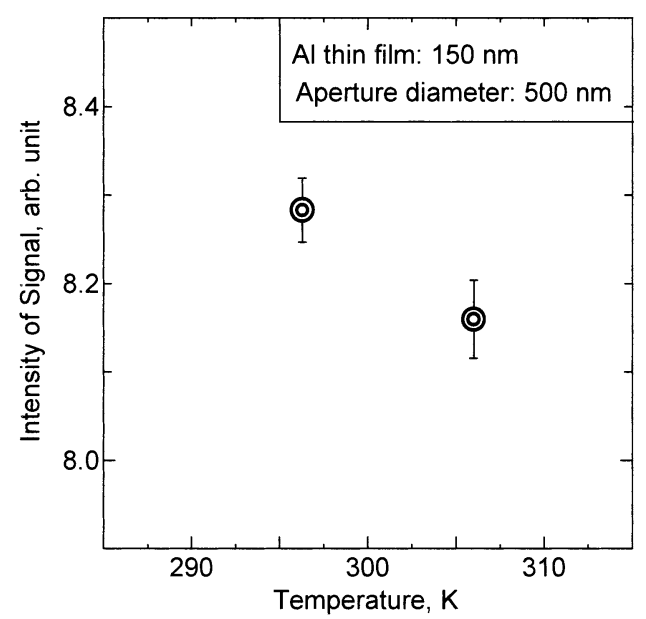

Fig. 9 The reflectivity changes corresponding to sample temperature. The sample is Al thin film on the BK7 substrate. The sample is illuminated by the near-field optics within a $250 \mathrm{~nm}$ radius.

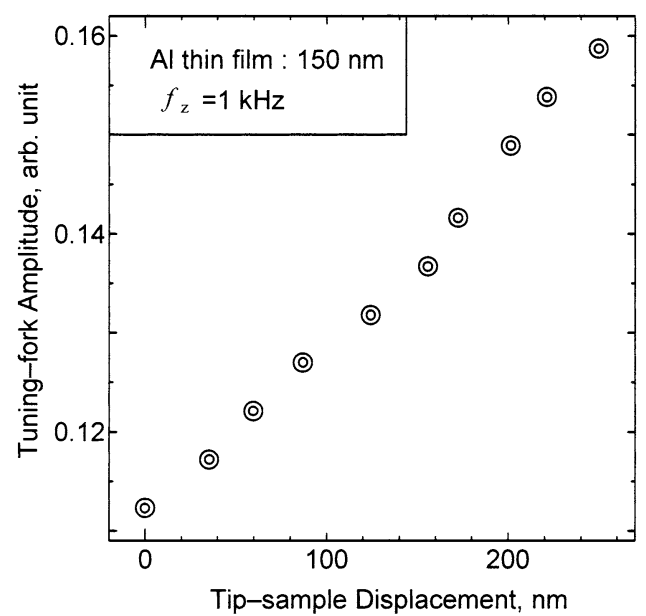

Fig. 10 The distance dependence of tuning-fork amplitude with probe oscillation method (POM). The signal is same as the normal mode without POM.

of near-field optics.

\subsection{Prototype of NOTN}

For the verification of the applicability of this technique to measure nanoscale thermal properties, the variation of reflectance in various temperatures is observed. Figure 9 compares the intensity of detected signal corresponding to sample temperature. An Al thin film on BK7 substrate is heated uniformly by a sheet heater, and the steady-state temperature distribution is detected as a thermoreflectance signal. By the theoretical estimation of reflectivity for $\mathrm{Al}$ thin film, the relative reflectance temperature coefficient $\beta$ is calculated as $6.54 \times 10^{-4} \mathrm{~K}^{-1}$ (vide Fig. 2). In the present work, the relative reflectance temperature coefficient is estimated to be $1.28 \times 10^{-3} \mathrm{~K}^{-1}$ by analyzing the slope in Fig. 9. Due to the thermal resistance between the thermocouple and sample, the measured temperature has a tendency to be lower than that of the pre- 
dicted value.

Finally, to confirm the capability to apply the present control system to POM (see section 5), the stability of the present system is evaluated. Figure 10 shows the distance dependence of shear-force, which is under POM. This result indicates that this system can control the tip-sample displacement under POM.

Although the measurement technique of NOTN is at an early stage, the validities of present apparatus, basic theory and proposed measurement theory are confirmed. The present study leads us to the conclusion that our prototype of NOTN has a potential of being applied to measure nanoscale thermophysical properties in-situ. A major task required to realize this potential is to check the capability of thermoreflectance method under POM.

\section{Acknowledgments}

This research was partially supported by the Ministry of Education, Science, Sports and Culture, Grant-in-Aid for No.14350112(B)(2), and Grant-in-Aid for Scientific Research, No.A39093.

\section{References}

( 1 ) Nagasaka, Y., Recent Development of Thermophysical Properties Research in Nano/Micro Scale, Proc. 23rd Jpn. Symp. Thermophys. Prop., (in Japanese), (2002), pp.268-270.

(2) Akabori, M., Sawajiri, O. and Nagasaka, Y., Thermal Diffusivity of Thin Films on a Substrate Measured by the Photoacoustic Method: Sputtered Ti and Zr Films, High Temp.-High Press, Vol.25 (1993), pp.411-415.

( 3 ) Cahill, D.G., Bullen, A. and Lee, S-M., Interface Thermal Conductance and the Thermal Conductivity of Multilayer Thin Films, High Temp.-High Press., Vol.32
(2000), pp.135-142.

( 4 ) Graebner, J.E., Jin, S., Kammlott, G.W., Herb, J.A. and Gardinier, C.F., Unusually High Thermal Conductivity in Diamond Films, Appl. Phys. Lett., Vol.60, No.13 (1992), pp.1576-1578.

( 5 ) Taketoshi, N., Baba, T. and Ono, A., Thermal Diffusivity Measurement of a Thin Metal Film with a Picosecond Thermoreflectance Technique, High Temp.High Press., Vol.34 (2002), pp.19-28.

(6) Shi, L., Kwon, O., Miner, A.C. and Majumdar, A., Design and Batch Fabrication of Probes for Sub-100 nm Scanning Thermal Microscopy, J. Microelectromech. Syst., Vol.10, No.3 (2001), pp.370-378.

( 7 ) Bethe, H.A., Theory of Diffraction by Small Holes, Phys. Rev., Vol.66, No.7 (1944), pp.163-182.

( 8 ) Durig, U., Pohl, D.W. and Rohner, F., Near-Field Optical-Scanning Microscopy, J. Appl. Phys., Vol.59, No.10 (1986), pp.3318-3327.

( 9 ) Ujihara, K., Reflectivity of Metals at High Temperatures, J. Appl. Phys., Vol.43, No.5 (1972), pp.23762383.

(10) Rosencwaig, A. and Gersho, A., Theory of the Photoacoustic Effect with Solids, J. Appl. Phys., Vol.47, No.1 (1976), pp.64-69.

(11) Okajima, T. and Hirotsu, S., Study of Probe-Surface Interaction in Shear-Force Microscopy: Effects of Humidity and Lateral Spring Constant, Opt. Rev., Vol.5, No.5 (1998), pp.303-309.

(12) Toledo-Crow, R., Yang, P.C., Chen, Y. and Vaez-Iravani, M., Near-Field Differential Scanning Optical Microscope with Atomic Force Regulation, Appl. Phys. Lett., Vol.60 (1992), pp.2957-2959.

(13) Martin, Y., Williams, C.C. and Wickramasinghe, H.K., Atomic Force Microscope-Force Mapping and Profiling on a Sub 100-Å Scale, J. Appl. Phys., Vol.61, No.10 (1987), pp.4723-4729. 